\title{
Earth history through the lens of carbonate diagenesis
}

Anne-Sofie C. AHM' AND John A. Higgins

\author{
Department of Geosciences, Princeton University, Princeton, \\ NJ 08544, USA (aahm@princeton.edu and \\ jahiggin@princeton.edu)
}

The chemistry of shallow-water carbonate sediments has been used to reconstruct the evolution of global biogeochemical cycles for a wide range of elements throughout Earth history. However, one of the main challenges to using this archive is the susceptibility of carbonate sediments to early diagenesis - the suite of reactions that occur as unlithified sediments are transformed into rocks. This talk will focus on extracting the primary chemical information from ancient carbonate sediments by improving our understanding of the early diagenetic process. By using a new geochemical framework that combines measurements of bulk carbonate calcium and magnesium isotope measurments, $\mathrm{Sr} / \mathrm{Ca}$ ratios, and a numerical model of carbonate diagenesis, it is possible to constrain the diagenetic process and thereby gain further insights into past surface conditions. This framework identifies carbonate end-members that have undergone either fluid-buffered or sedimentbuffered diagenesis and thereby extracts information about the diagenetic fluid and the primary carbonate sediment. This framework has been tested in recent carbonate sediments from the Bahamas, showing that the geochemical variability in calcium, magnesium, and carbon isotopes can be explained by the combined effects of carbonate mineralogy (aragonite, calcite, dolomite) and early marine diagenesis. Moreover, we use this geochemical framework to reinterpret the chemistry of ancient carbonate sediments throughout Earth history going back to the Archean where geochemical changes often have been attributed to perturbations in the global carbon and oxygen cycles. Finally, when applied to a wide range of geochemical proxies in ancient shallow-marine carbonate sediments (e.g., lithium, strontium, and sulfur isotopes), this geochemical framework can be used to create more robust records of past seawater chemistry. 